NP11 (continued)

Description: Mi Niño was developed to capture "parentcentered" and "child-centered" food-related behaviors with items based on the previous English language My Child at Mealtime tool, and tailored through cognitive interviews for low-income Spanish audiences. Factor analysis will be conducted and reliability of the resulting scales tested. Mealtime observations will be used to assess the accuracy of item responses to observed mealtime behaviors.

Evaluation: Mi Niño was completed by 205 Hispanic parents from WIC or Head Start. Exploratory factor analysis of the responses will be conducted to examine whether the responses correspond to the "parent-centered" and "child-centered" subscales of MCMT. A subsample $(n=60)$ completed mealtime videotaping in their homes. All videotapes were coded by two independent coders for parent food-related behaviors using Noldus v.10 for the following behaviors: physical manipulation, feeding child, verbal demands to eat, bargaining; eating statements, eating inquiries, requests to eat, preference statements about food, and general food statements. Inter-rater reliability was established between coders through double-coding of $40 \%$ of coded videotapes (Cohens kappa $>.70$ ). All discrepancies (coded outside of a five-second window of each other or application of different codes) between the two coders on the shared videotapes were resolved through discussion and consensus. The total number of occurrences of each code was exported to SPSS v.22 and then adjusted for the length of the meal, resulting in a rate per minute (RPM) for each behavior. Pearson's correlations will examine whether rate of the observed behaviors corresponds to the behavior rating on the Mi Ninos scale.

Conclusion and Implications: Parent food-related behaviors are related to the development of obesogenic behaviors in children. Validation of a Spanish language self-assessment tool to capture these behaviors would be a valuable tool to help programs effectively target them for obesity prevention.

Funding: 2015-68001-23280.

\section{NP12 Guided Goal Setting Behaviors of Spanish and English-Speaking EFNEP Participants in a Medical Clinic Setting}

Mical Shilts, PhD, shiltsm@csus.edu, California State University, Sacramento, 9798 Sun Kosi Way, Elk Grove, CA 95757; Abbey Lake, California State University, Sacramento; Cherae Washington, California State University, Sacramento; Larissa Leavens, BS, University of California, Davis; Karina Diaz Rios, PhD, RD, University of California, Merced; Lenna Ontai, PhD, University of California, Davis; Katherine Panarella, MPH, MS, University of California Cooperative Extension Agricultural and Natural Resources; Dennis Styne, MD, University of California, Davis; Marilyn Townsend, PhD, RD, University of California, Davis

Objective: To address pediatric obesity risk reduction, EFNEP was embedded within medical clinics where pediatricians referred parents to the intervention. The traditional EFNEP intervention was augmented with guided goal setting (GGS), Healthy Kids (HK) obesity risk assessments, motivational modeling, and parenting topics. This investigation focused on assessing goal-setting behaviors of EFNEP participants attending the medical clinic intervention.

Description: Pediatricians referred 686 parents (30\% Spanish speaking) to the EFNEP intervention. More than one-third ( $n=264)$ of parents referred expressed interest in attending with 195 verbally agreeing to enroll in the intervention anchored with GGS. GGS gives goal choices from a collection of practitioner developed major and minor goals so parents can make their own goal decisions. Parents completed the HK assessment tools during session one, they were guided to select nutrition and parenting minor goals at session two, but at subsequent sessions, were given the options to continue with the same goal, select a new guided goal, modify the existing goal, or create a new goal. Goal selections, effort, and attainment were tracked weekly.

Evaluation: Twenty intervention series with parents or caregivers ( $\mathrm{n}=105,50 \%$ Spanish speaking) were conducted at five medical clinics with $78 \%$ of parents completing four or more sessions. All parents engaged in GGS with most making nutrition and parenting goal effort and achievement (Spanish 96\%, 74\%; English 92\%, 69\%, respectively). Parents selected fruit and vegetable goal topics frequently and rarely chose contingency management topics. Most parents selected a new goal each session with Spanish speaking parents more likely to select a guided option ( $72 \%$ vs $61 \%$ ).

Conclusion and Implications: Parents referred by their child's pediatrician attended the EFNEP intervention and engaged in the GGS activities. Parents reported a high level of goal commitment with Spanish speaking parents attending more classes and reporting higher levels of goal effort compared to English speaking parents. GGS was shown to be a suitable behavioral strategy for Spanish and English speaking parents attending EFNEP within a medical clinic setting.

Funding: 2015-68001-23280.

\section{NP13 Designing Retrospective Evaluation Tools with Low-Literacy Adults: Development Process}

Marilyn Townsend,PhD, RD, mstownsend@ucdavis.edu, University of California, Davis, Department of Nutrition, 3135 Meyer Hall, One Shields Ave, Davis, CA 95616-5270; Mical Shilts, PhD, California State University, Sacramento; Karina Diaz Rios, PhD, RDN, University of California, Merced; Larissa Leavens, BS, University of California, Davis; Katherine Panarella, MPH, MS, University of California Cooperative Extension Agricultural and Natural Resources

Objective: Collecting 'before' and 'after' ratings at the time of post-testing for program evaluation, reduces the likelihood of response-shift bias with a resulting increase 